Original article

\title{
Optimization of dental implant treatment
}

\author{
Dmitriy V. Ivanov ${ }^{1}$, Aleksandr V. Dol ${ }^{1}$, Dmitriy A. Smirnov ${ }^{2}$ \\ ${ }^{1}$ Saratov State University, Saratov, Russia \\ ${ }^{2}$ Vash Doktor LTD, Saratov, Russia
}

Received 8 December 2015, Accepted 30 December 2015

(C) 2015, Ivanov D.V., Dol A.V., Smirnov D.A

C 2015, Russian Open Medical Journal

\begin{abstract}
Aim - Modern dentistry cannot exist without dental implantation. The lifetime of the installed implants depends on condition of the bone and on the quality of the treatment planning and surgery technique. Usually, complications during the implant treatment are related to the inability to accurately predict the condition and location of intraosseous structure that entails the selection of the wrong type of implant and installation position.

Methods - This work is devoted to the "bone-implant" system investigation aiming on the optimization of dental prostheses installation. The objective of this study was to develop the implant treatment planning technique. Modern non-invasive methods such as computer tomography (CT) and 3D-scanning as well as numerical calculations and 3D-prototyping allow optimizing all of dental prosthetics stages.

Results - In this work, complex methodology of dental prosthesis installation is presented. Patient-specific dental templates developed in this work are easy to create and very accurate.

Conclusion - CT processing method, numerical modeling and 3D prototyping techniques allowed us to develop a methodology of dental prosthesis installation. An integrated approach to the planning of implant surgery can significantly reduce the risk of complications in the first few days after treatment, and throughout the period of operation of the prosthesis.
\end{abstract}

Keywords: dental prosthesis, implant, bone, modeling, 3D-model, dental template

Cite as Ivanov DV, Dol AV, Smirnov DA. Optimization of dental implant treatment. Russian Open Medical Journal 2016 ; 5: e0102.

Correspondence to Dmitriy Ivanov. Address: 83, Astrakhanskaya str., Saratov, 410012, Russia. Phone: +7 917 3146807. E-mail: ivanovdv@gmail.com

\section{Introduction}

According to statistics, in Russia people over 40 years old in $90 \%$ of cases have at least one lost tooth (Figure 1) [1]. In this regard, modern dentistry cannot exist without the dental prosthesis. Durability of implants depends on both the general condition of the patient's mouth tissues and quality of the preparatory stage and the surgeon qualification.

Moreover, in $10 \%$ of cases due to improper installation of implants repeated surgery is required. Additional surgeries are often required during the bone tissue preparation process before the implantation, which increases the invasiveness of the method and leads to a significant increase in the time required to complete treatment.

Clearly, there is a need to optimize the whole process of implantation process to reduce the number of surgical complications and minimize the number of surgery stages. It is possible to avoid unsuccessful surgery operations with the help of modern biomechanical and mathematical methods, which use three-dimensional computer design and high-precision numerical calculations. In this case, at the planning stage of the treatment we should take into account special structure of the bone tissue of each patient. Prognosis of the surgical operation results can be made according to the biomechanics [2] that involves analysis of stress-strain state of the bone-implant system. Surgeon can choose the optimal location of the implant based on the computer modeling results. Patient-specific plastic dental template can be produced with help of $3 \mathrm{D}$ printer at the final stage of the preparation procedure.

\section{Material and Methods}

Computer tomography (CT) scans of patients who underwent implant treatment in dental clinic "Vash Doctor" (Saratov, Russia) were used as initial data in modeling process. Additionally, the results of oral 3D scanning of the same patients were used.

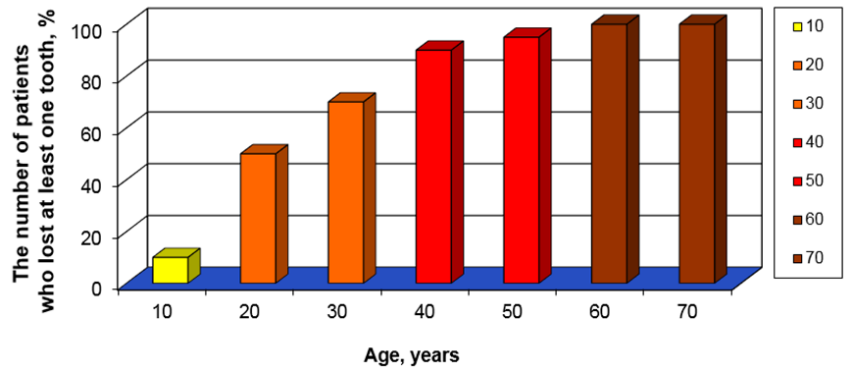

Figure 1. Statistical data of the Russian Federation residents who have a need for prosthetics (according to Russian Federal State Statistics Service). 

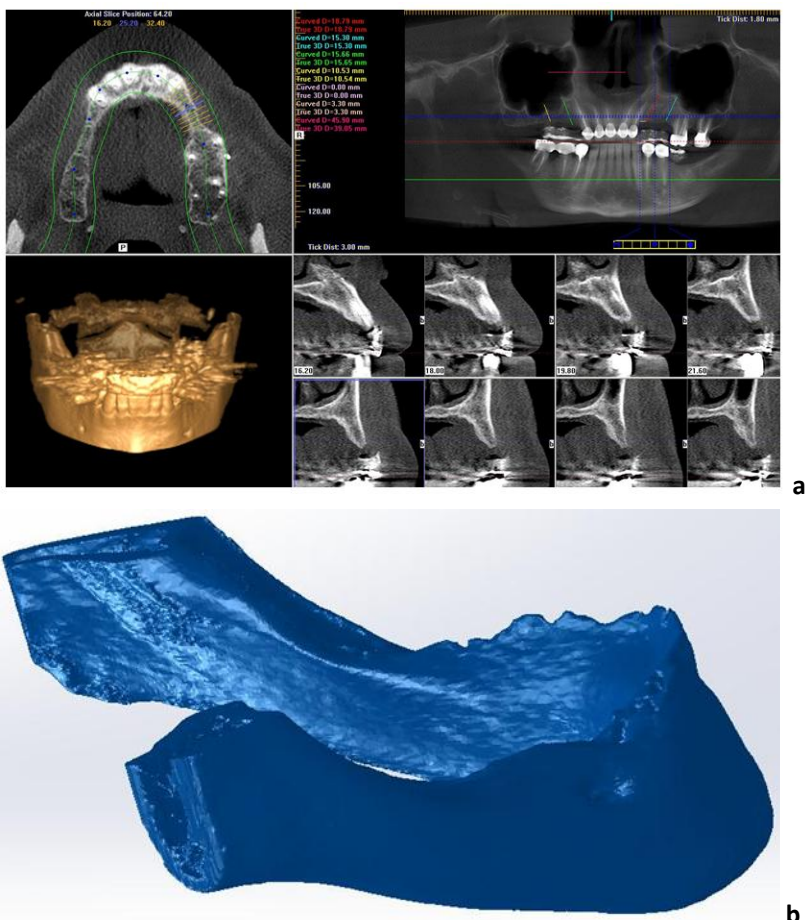

Figure 2. Processing CT scan of jaw and the construction of its 3D model: $a$ - patient's jaw CT, b-3D model of jaw.

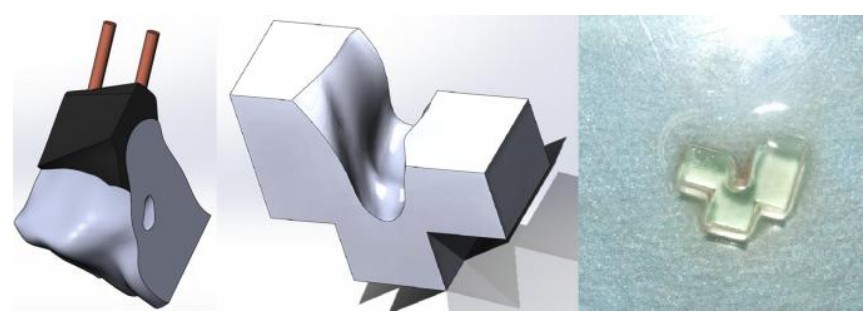

Figure 3. Positioning of the implants in the bone and a template creation: implants and the template in the jaw (left - a), 3D model of the surgical template (center $-b)$, plastic surgical template $($ right $-c)$.

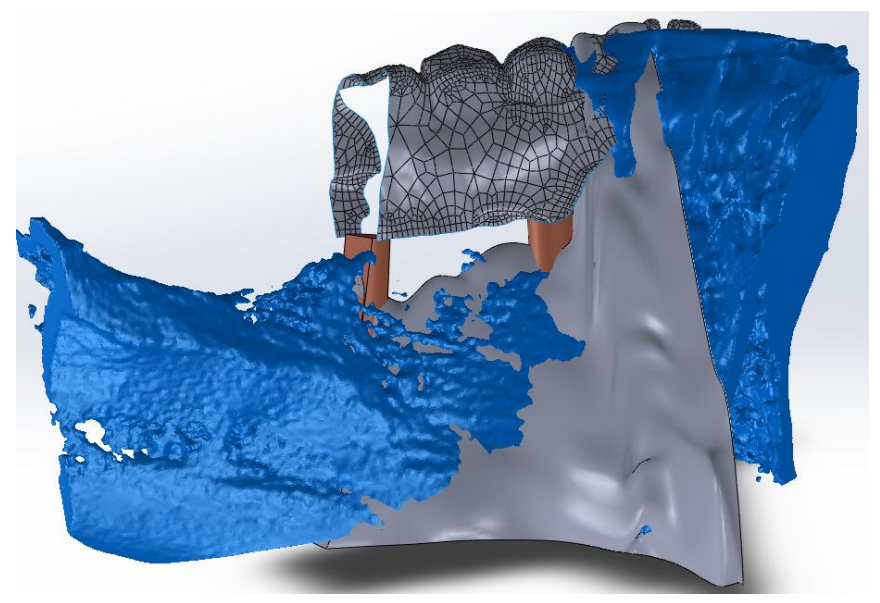

Figure 4. Combined 3D models of human teeth and jaw.

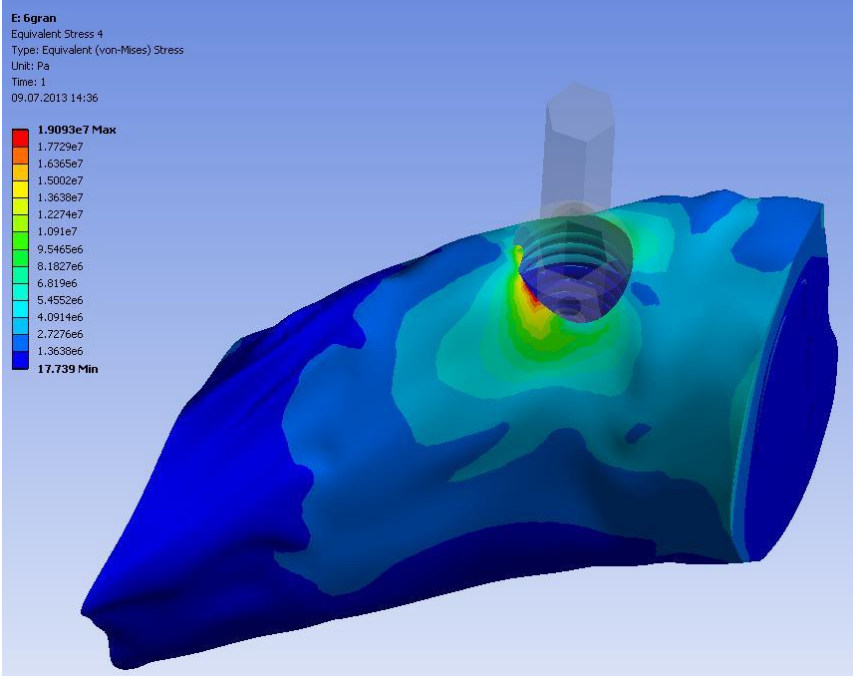

Figure 5. Effective stress distribution in the model of the implant and part of the jaw.

Initially, CT images were processed in the ICatVision software (Imaging Sciences International, LLC, USA), further cross sections were passed into computer-aided design software SolidWorks (Dassault Systèmes SolidWorks Corporation, USA). SolidWorks was used to create 3D models of the jaws and for the combination of these models with 3D images of the oral cavity. SolidWorks was also used for creating implant 3D models, placement of implants and creating models of dental templates.

Bone-implant-prosthesis systems under external chewable loads were investigated in finite-element software Ansys (Ansys Inc., USA). Contact interaction between jaw, implants and prosthesis was simulated as stationary process. The lower part of jaw was constrained and $100 \mathrm{~N}$ loads at 30 degrees to the axis of the implant were applied to the prosthesis.

There are a number of studies [3, 4], showing that bone density, calculated using the Haunsfild number have a good correlation with data calculated using bone densitometry (DEXA). Thus calculated bone density allows obtaining the bone elastic modulus (Young's modulus) required for performing numerical calculations. So in this work mechanical properties of bone tissues were calculated individually for each patient using Hounsfield number.

\section{Results}

In this paper, the basic stages of the implantation process optimization are presented. These stages are covering the whole treatment process from the first patient's visit to the final surgery.

First of all, it is necessary to conduct an initial definition of the implants location and angles of inclination (Figure 2a). This could be made with the help of patient's CT data. Then accurate threedimensional model of the jaw should be constructed based on CT data (Figure 2b) [5]. It should be noted that during the construction of the model it is necessary to accurately select a range of grey color on CT scan. Error in the choice of the range may result in "loss" of bone parts or, on the contrary, in the appearance of unnecessary elements and tissues. This could have a negative impact on the quality of the calculations in the future. 


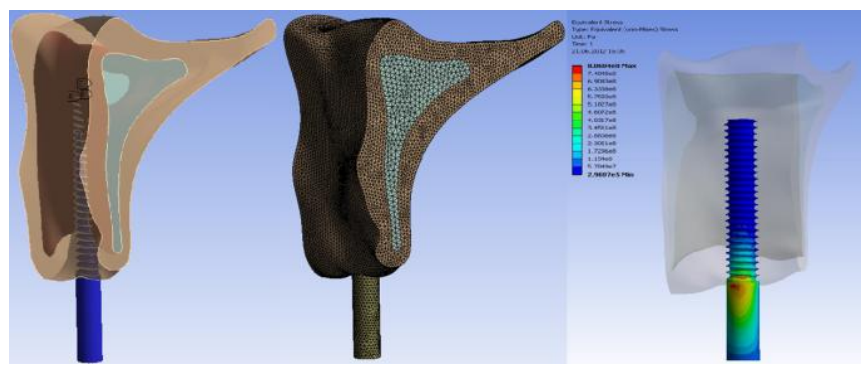

Figure 6. Main stages of the finite-element calculation: creation of 3D geometry (left), finite element mesh creation (center), analysis of the numerical results (right).
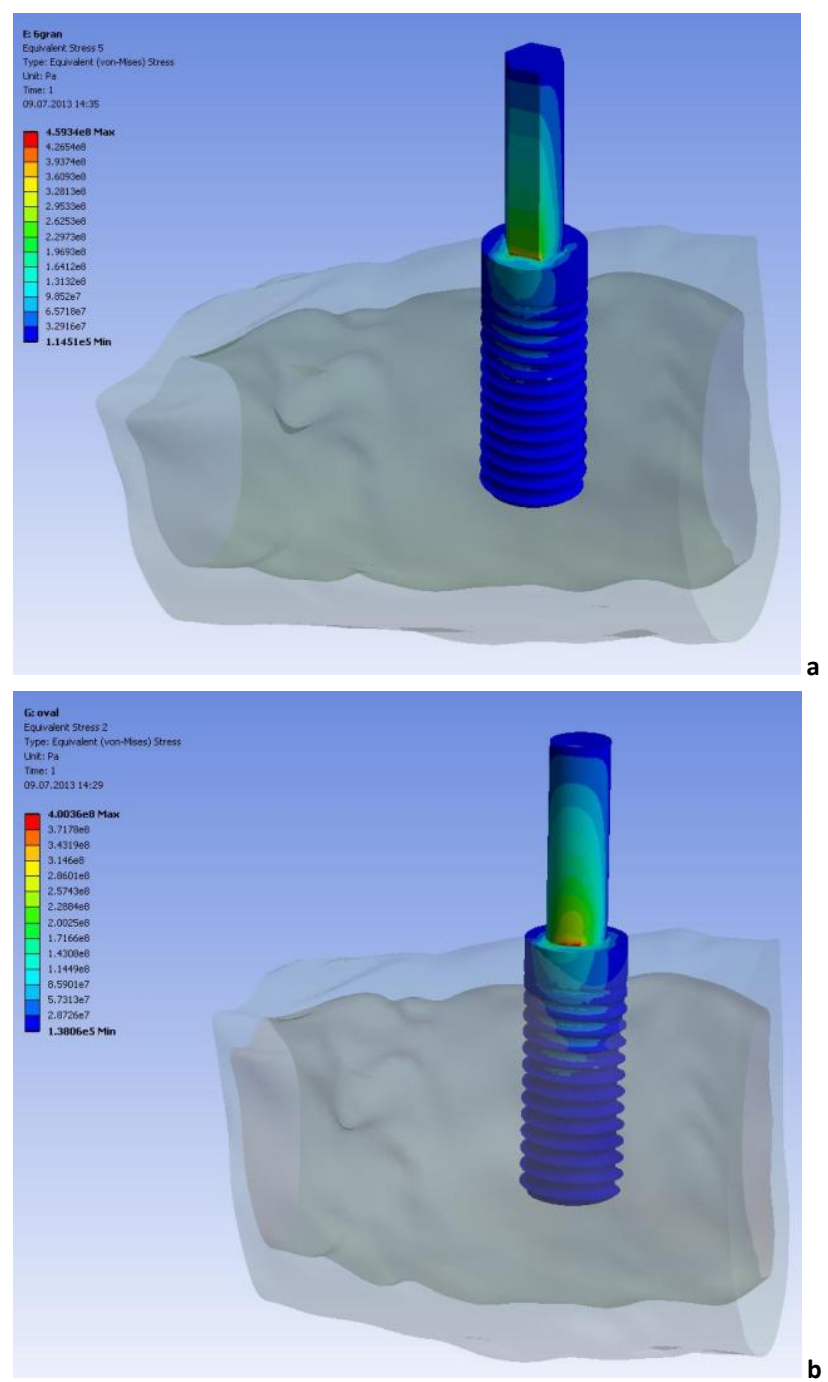

Figure 7. Effective stress distribution in different types of abutments: a hexagonal abutment; b - oval abutment.

The constructed 3D model of the jaw allows surgeon to positon implant models with a high degree of accuracy (Figure 3a). After virtual implantation procedure, a model of dental template can be constructed on the relevant bone segment (Figures $3 a$, and $3 b)$. This template further should be made with the help of $3 D$ printing technique and could be used in dental surgery for implant placement.

More accurate positioning of implants can be carried out if the results of CT scan of the jaw would be combined with the results of 3D scan the human oral cavity. As a result of 3D scanning we can build 3D model of human teeth and soft tissues. Figure 4 shows the combined model of human teeth (grey color with black edges), jaw (blue color) and implants (brown color).

Implant surgery should be prepared not only from medical, but also from a mechanical point of view. This means that the installed structures must be placed in such a way to avoid stress concentrations in bone and implants for all types of chewing loads. Stress concentrations lead to metal fatigue and implant failure. Stresses, which arise in implants and bone, should not reach critical stress values for implant and bone material. Finite-element numerical calculations allow solving the problem that is connected with high stress values and stress concentrations (Figure 5).

Numerical modeling of the bone-implant-prosthesis system requires the construction of 3D model of bone and its combination with an implant and prosthesis models. Next computational finiteelement mesh should be constructed and the stress-strain state of the investigated system could be then studied (Figure 6) [6].

\section{Discussion}

Optimization of implant treatment in the last decade has received increasing attention because the method of implants installation has showed itself as one of the most popular and widely used in clinical practice since its inception 40 years ago. Thus, the issue of improving the quality of treatment is not only to optimize the shape of the implant, but also to place the implant in the right location and at the correct angle.

There are a number of articles that are dedicated to the studying of the interaction between bone and short abutments (shorter than $9 \mathrm{~mm}$ ) [2, 7] and compared with longer models [8, 9]. Most researchers agree that the stress distribution in the bone in case of short implants in almost all parameters respects similar to the results that show standard samples. However, for certain types of loads short implants may break off or seriously damage the surrounding bone due to the stress concentration zones in the contact area between implant and bone.

According to the authors, the choice of a particular implant plays the main role in the future work of the whole structure and should be well-founded. When choosing an implant for a particular patient surgeon should take into account the types of loads that will affect the prosthesis during its operation in a particular installation zone [10].

Our numerical calculations revealed no significant differences between short and standard implants. However, similar to other studies [8], numerical simulations showed that two implants are much more stable hold the tooth than one implant.

A number of numerical calculations to optimize the shape of the abutment and to choose the length of the implant were held by authors to date and are presented in this article. Calculations have shown that in terms of mechanics oval abutments (Figure $7 \mathrm{~b}$ ) showed the best results. In such constructions, unlike the hexagons (Figure 7a) or a hexagon with rounded corners, we did not obtain stress concentration zones, which can lead to metal fatigue. Furthermore, oval abutments were more stable in case of torsional loading than round abutments. 
Templates proposed in the article fully comply with the patient's bone and allow surgeon to orient the implant during operation. The surgeon only needs to impose template on the bone, drill a hole and then remove the template and set the implant. This significantly improves the accuracy of implant positioning in natural tissues structure. Moreover, during the treatment only one incision of the gums should be performed.

These templates are also intended to be used in operations aimed on bone building. In this case, template will be made of biocompatible and biodegradable materials (for example, polylactide (PLA)-plastic that is used in 3D printing technology). Bone tissue building is a process that is directly related to dental implantation. In fact, the prolonged absence of teeth leads to bone loss due to the lack or absence of a functional load. Lack of bone can be caused by individual characteristics of a person. A sufficient amount of bone is needed for the proper implant installation. It could be obtained by increasing bone tissue with the help of the bone graft installation or the use of the individual biodegradable template.

Surgical templates offered in this paper do not require additional operations at manufacturing stage and can be grown with the help of 3D printer within one business day from the date of patients visit.

\section{Conclusion}

An integrated approach to the planning of implant surgery can significantly reduce the risk of complications in the first few days after treatment and throughout the period of the prosthesis. Numerical calculations at the preparatory stage allow predicting the behavior of the "bone-implant" system under the influence of various types of chewing loads. Thus, surgeon can obtain the position of the implant in advance, ensuring its optimal position. Moreover, in the framework of this method a surgical template should be created. This template allows placing the implant exactly as it was planned in the preparatory phase, which greatly simplifies the work of surgeon.

\section{Acknowledgments}

The work was supported by the Foundation for Assistance to Small Innovative Enterprises in Science and Technology (U.M.N.I.K.).

\section{Conflict of interest}

There is no any conflict of interest.

\section{References}

1. Smirnov DA, Ivanov DV, Dol AV. Use of computer-aided design software to build a 3d model of the jaw. In: Proceedings of All-Russian Conference of Young Scientists with international participation "Applied Biomechanics". Saratov, Russia, 2015: 138-141. Russian

2. Hasan I, Bourauel C, Mundt T, Heinemann F. Biomechanics and load resistance of short dental implants: a review of the literature. ISRN Dent 2013; 8: 424592. (doi: 10.1155/2013/424592) (PMID: 23738085)

3. Khan SN, Warkhedkar RM, Shyam AK. Analysis of Hounsfield unit of human bones for strength evaluation. Procedia Materials Science 2014; 6: 512-519. (doi: 10.1016/j.mspro.2014.07.065)

4. Lee S, Chung CK, Oh SH, Park SB. Correlation between bone mineral density measured by dual-energy $\mathrm{X}$-ray absorptiometry and Hounsfield units measured by diagnostic CT in lumbar spine. J Korean Neurosurg Soc 2013; 54(5): 384-389. (doi: 10.3340/jkns.2013.54.5.384) (PMID: 24379944)
5. Ivanov DV, Lepilin AV, Smirnov DA, Dol AV. The possibility of different CAD systems in the construction of mathematical model of bone tissue. Saratov Journal of Medical Scientific Research 2013; 9(3): 403405. Russian

6. Smirnov DA, Lomakin MV, Lepilin AV, Ivanov DV, Dol AV. Biomechanical study of the stress-strain state of the bone-implantabatement system in the region of short dental implants. Russian Stomatology 2013; 6(1): 21-24. Russian

7. Mandhane SS, More AP. A review: Evaluation of design parameters of dental implant abutment. International Journal of Emerging Science and Engineering 2014; 2(4): 64-67.

8. Shrikar RD, Singh R, Karthikeyan I. 2D FEA of evaluation of micromovements and stresses at bone-implant interface in immediately loaded tapered implants in the posterior maxilla. J Indian Soc Periodontol 2013; 17(5): 637-643. (PMID: 24174759) (doi: 10.4103/0972-124X.119283)

9. Lin C-L, Kuo Y-C, Lin T-S. Effects of dental implant length and bone quality on biomechanical responses in bone around implants: a 3-D non-linear finite element analysis. Biomed Eng Appl Basis Commun 2005; 17(1): 44-48. (DOI: 10.4015/S1016237205000081)

10. Brunski JB. Biomechanical factors affecting the bone-dental implant interface. Clin Mater 1992; 10(3): 153-201. (PMID: 10149982)

\section{Authors:}

Dmitriy Ivanov - PhD, Deputy Head of Department, Educational-Research Institute of Nanostructures and Biosystems, Saratov State University, Saratov, Russia.

Aleksandr Dol - PhD, Lead Coder, Educational-Research Institute of Nanostructures and Biosystems, Saratov State University, Saratov, Russia.

Dmitriy Smirnov - PhD, Head of dental clinic "Vash Doktor", Saratov, Russia. 